\title{
Application of thermoelastic stress analysis for the experimental evaluation of the effective stress intensity factor
}

\author{
Francisco A. Díaz \\ Universidad de Jaén, Departamento de Ingeniería Mecánica y Minera, Campus las Lagunillas, Edificio A3, 23071, Jaén, Spain
}

Eann A. Patterson

University of Liverpool, School of Engineering, Harrison Hughes Building, The Quadrangle, Liverpool L69 3GH, UK

John R. Yates

University of Manchester, School of Mechanical, Aerospace and Civil Engineering, George Begg Building, Manchester, M60 $1 Q D, U K$

\begin{abstract}
In recent years, the advent of staring array detectors has made Thermoelastic Stress Analysis (TSA) a technique with considerable potential for fatigue and fracture mechanics applications. The technique is noncontacting and provides full field stress maps from the surface of cyclically loaded components. In addition, the technique appears to have a great potential in the evaluation of the effective stress intensity factor range during fatigue since fracture mechanics parameters are derived directly from the temperature changes in the vicinity of the crack tip rather than from remote data. In the current work TSA is presented as a novel methodology for measuring the effective stress intensity factor from the analysis of thermoelastic images. $\Delta K$ values inferred using TSA have been employed to estimate an equivalent opening/closing load at different R-ratios in a cracked aluminium 2024 CT specimen. Results have been compared with those obtained using the strain-offset technique showing a good level of agreement.
\end{abstract}

KEYwORDS. Thermoelastic Stress Analysis (TSA); Fatigue; Effective Stress Intensity Factor.

\section{INTRODUCTION}

$\mathrm{F}$ atigue cracks have been one of the main sources of structural failures in machines for two centuries. The application of fracture mechanics to engineering design has led to more efficient use of structures and components, which leads to great economic benefits by avoiding premature retirement of serviceable machines. However, there are still some aspects of fatigue that remain partially understood, such as the crack closure effect. This lack of understanding arises principally from the difficulties associated in quantifying the phenomenon and measuring its effect on the crack driving force [1].

In recent years, advances in infrared thermography together with the development of infrared staring array radiometer detectors have made it possible to apply this technology to fatigue damage assessments. Such an example is Thermoelastic Stress Analysis (TSA). This experimental technique makes it possible to infer the in-plane stresses on a solid structure by measuring the small temperature changes induced as a result of a cyclic load.

From the fatigue point of view, TSA constitutes a breakthrough over other experimental stress analysis techniques. With TSA the stress intensity factor is directly obtained by computing the cyclic stress field ahead of the crack tip, which makes it possible to evaluate the actual crack driving force for the fatigue advance [ 2 and 3$]$. The outcome is that the TSA 
method provides a direct measurement of the effective $\Delta K$ that is usually measured indirectly by compliance techniques. This arises from the fact that with TSA the near crack tip stress distribution is obtained from the temperature variations at the specimen surface as a result of the thermoelastic effect, providing a direct assessment of the cyclic strains in the field around the crack tip. Hence, the observed crack tip stress pattern is the result of the specimen's response to the applied loading cycle. To support the idea that TSA can provide accurate information about the real fatigue crack driving force, a set experiments using aluminium $2024 \mathrm{CT}$ specimens have been conducted. As a result, $\Delta K$ results obtained using thermoelastic images have been employed to infer an equivalent value for the opening/closing load for increasing $R$ ratios. Results have been compared with those obtained using the strain offset technique, showing in all cases a good level of agreement, highlighting the value of TSA for fatigue damage assessment.

\section{PHYSICAL PRINCIPLES OF THERMOELASTIC EFFECT}

$\mathrm{T}$ he thermoelastic effect was first reported by Lord Kelvin [4] in 1853. It states that any substance in nature experiences changes in its temperature when its volume is changed due to the application of a force: compressive loads cause an increase in temperature while tensile load produces a decrease in temperature. Consequently, if a cyclic load is applied to a component there will be a cyclic change in temperature. Under elastic conditions, these temperature variations are normally quite small (tens of $\mathrm{mK}$ ) and they are normally ignored in the classical theory of elasticity. However, with the use of high precision infrared detectors these temperature changes can be measured.

The thermoelastic effect is a reversible conversion between the mechanical and thermal forms of energy, since the temperature variation will reverse when the load is withdrawn. However, this energy conversion is reversible only if the elastic range of the material is not exceeded and there is no significant transport of heat during loading and unloading of the structure. Moreover, thermoelastic theory states that under adiabatic and reversible conditions, the temperature variations experienced by the cyclically loaded material are proportional to the sum of principal stresses. The relation between the change in temperature due to the application of a cyclic loading and the stress range of a linear elastic and homogeneous material can be written as:

$$
\Delta \mathrm{T}=\frac{-\alpha \mathrm{T}}{\rho \mathrm{C}_{\mathrm{p}}} \Delta\left(\sigma_{1}+\sigma_{2}\right)
$$

Where $\alpha$ is the coefficient of thermal expansion, $T$ is the absolute temperature of the material, $\rho$ is the density, $C_{p}$ is the specific heat at constant pressure and $\sigma_{1}$ and $\sigma_{2}$ are the principal stresses.

To ensure that the experimentally recorded variation is linear, the load cycle must be fast enough to prevent heat transport and thus achieve adiabatic conditions. Truly adiabatic conditions may be achieved only if the thermal conductivity of the material is zero or no stress gradients are present in the specimen. However, if the load frequency is high enough the thermal diffusion length is reduced and the presence of non-adiabatic effects is minimized.

In modern TSA, an infrared camera based on a staring array of photon detectors is used to measure the temperature changes at the surface of the component as a consequence of an applied cyclic load (figure 1A). The technique measures load-correlated temperature signals in a cyclically loaded body using infrared detectors. Thus, the analysis of thermoelastic response has to be done under dynamic conditions at an adequate frequency to ensure adiabatic conditions in order to prevent heat transfer through the test piece. When adiabatic conditions are achieved and maintained during the test, the relation between the induced temperature change and the change in the sum of principal stresses is assumed to be linear, and thus the variation in the sum of principal stresses can be experimentally inferred by processing the thermoelastic signal according to the following equation:

$$
\Delta\left(\sigma_{1}+\sigma_{2}\right)=\Delta\left(\sigma_{\mathrm{x}}+\sigma_{\mathrm{y}}\right)=\frac{\mathrm{E}}{1-v} \Delta\left(\varepsilon_{\mathrm{x}}+\varepsilon_{\mathrm{y}}\right)=\mathrm{A} \mathrm{S}
$$

Where, $E$, is the Young's modulus, $v$, is the Poisson's ratio, $\varepsilon_{x}$ and $\varepsilon_{y}$, are the strains in two orthogonal directions, $S$, is the thermoelastic signal and $\mathrm{A}$ is a calibration constant.

To translate the thermal units into stresses a calibration process needs to be performed [5]. This process consists essentially in defining the stress at a point in the images for a given load on the structure. A common method used for calibration consists of generating an independent measure of stress using strain gauges as illustrated in figure 1.B. 
A)
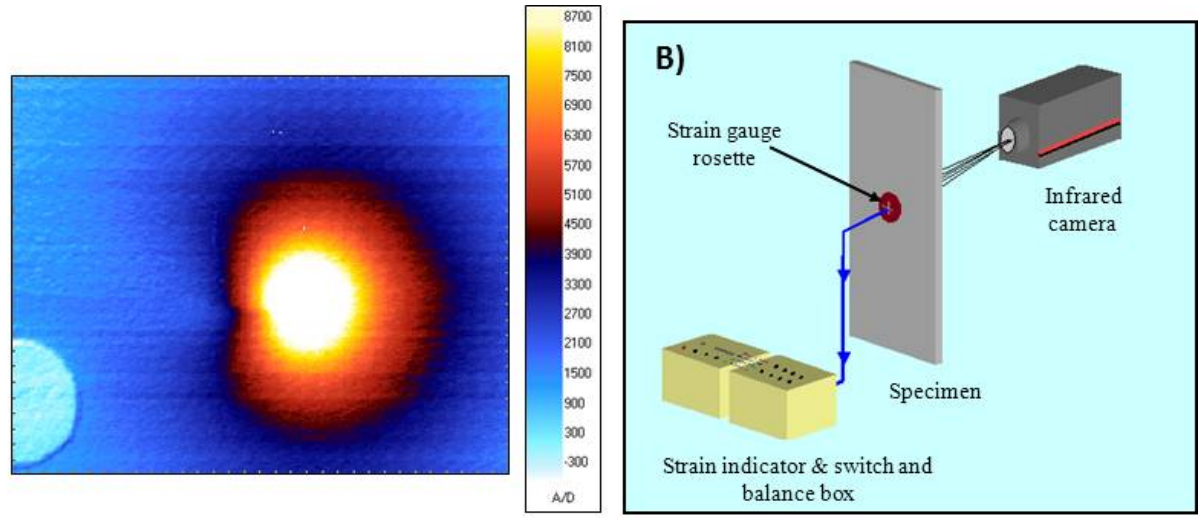

Figure 1: A) Typical thermoelastic pattern of a fatigue crack. B) Schematic illustration of the calibration process.

\section{PHASE SHIFT INFORMATION AT THE CRACK TIP REGION}

$\mathrm{T}$ hermoelastic information is normally presented as a vector where the modulus is proportional to the change in temperature experienced by the specimen due to the thermoelastic effect and the phase denotes the angular shift between the thermoelastic and the reference signals (loading signal). Thus, the magnitude of the phase is normally constant unless adiabatic conditions are not achieved.

However, there are two phenomena that lead to a lack of adiabatic conditions and hence a change in phase: heat generation due to plastic work and the presence of high stress gradients. Both are conditions that occur near the crack tip region. In these circumstances heat conduction start taking place blurring the data at those regions, and the direct observation of the crack tip from a thermoelastic image is difficult.

For image processing purposes, the reference and the thermoelastic signals are set in-phase. Thus, the phase map should be zero in all those points where adiabatic conditions are achieved. However, near the crack tip, plasticity and high stress gradients lead to a loss of adiabatic conditions. This loss of adiabaticity can be easily identified in the phase map (figure 2.A).

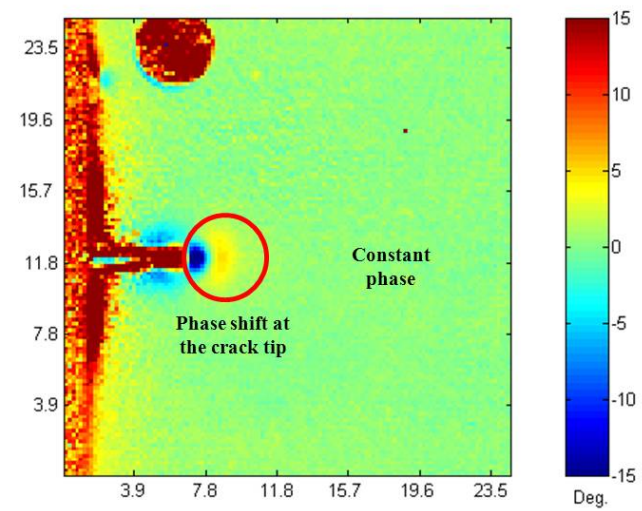

Figure 2: Typical phase map for a $6 \mathrm{~mm}$ fatigue crack. Illustration of the phase shift occurring at the crack tip as a result of the loss of adiabatic conditions (dimensions in $\mathrm{mm}$ ).

\section{METHODOLOGY FOR CALCULATING THE STRESS INTENSITY FACTOR RANGE FROM THERMOELASTIC IMAGES}

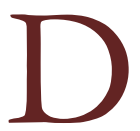

ifferent approaches have been developed in recent years as general methods for calculating the SIF from the analysis of thermoelastic images. Some of these approaches act by fitting a mathematical model describing the near crack tip stress field to a set of experimental data points collected at the near crack tip region [2 and 3]. As a result of the mathematical fitting the SIF can be inferred. The adopted method for calculating the SIF [2] is based on a 
Multi- Point Over-Deterministic (MPOD) method [6] in conjunction with a description of the near crack tip stress field based on Muskhelishvili's complex potentials [7].

Direct observation of the crack tip is often difficult since data very near the crack tip tend to be blurred as a result of plasticity and the presence of high stress gradients. To calculate $\Delta K$ using TSA, it is required to obtain a thermoelastic map of the region near the crack tip. Subsequently, a set of data points has to be collected in the region surrounding the crack tip. In this sense care must be taken to avoid collecting data points at those locations affected by near crack tip plasticity. To identify such a region Stanley's methodology has been employed [3]. Stanley's method [8] combines equation 2 with a mathematical expression describing the crack stress sum derived from Westergaard's model [9].

$$
\Delta\left(\sigma_{\mathrm{x}}+\sigma_{\mathrm{y}}\right)=\Delta\left(\sigma_{1}+\sigma_{2}\right)=\mathrm{A} \mathrm{S}=\frac{2 \Delta \mathrm{K}_{\mathrm{I}}}{\sqrt{2 \pi \mathrm{r}}} \cos \left(\frac{\theta}{2}\right)-\frac{2 \Delta \mathrm{K}_{\mathrm{II}}}{\sqrt{2 \pi \mathrm{r}}} \sin \left(\frac{\theta}{2}\right)
$$

For the case of pure mode I cracks, Stanley observed that a maximum thermoelastic signal, $S_{\text {max }}$, along any line parallel to the crack, occurred at a $60^{\circ}$ angle with respect to the crack. Taking this into account, equation 3 can be rearranged into a linear equation relating the vertical distance from the crack to any parallel line with the inverse square of the maximum thermoelastic signal along that particular line, $1 / S^{2} \max$.

$$
\mathrm{y}=\left(\frac{3 \sqrt{3} \Delta \mathrm{K}_{\mathrm{I}}^{2}}{4 \pi \mathrm{A}^{2}}\right) \frac{1}{\mathrm{~S}_{\text {max }}^{2}}
$$

If the previous relation for a real thermoelastic image is plotted (Figure 3), three different regions can be identified:

1. Region A. In this region no linear behaviour is observed since there is a loss of adiabatic conditions due to high stress gradients and crack tip plasticity.

2. Region B. In this region a clear linear behaviour is observed. This can be employed to defined the region of validity of the model since the same behaviour as described by equation 6 is observed.

3. Region C. In this region there is a deviation from the linear behaviour observed in region $\mathrm{B}$, which indicates inappropriate use of this mathematical model.

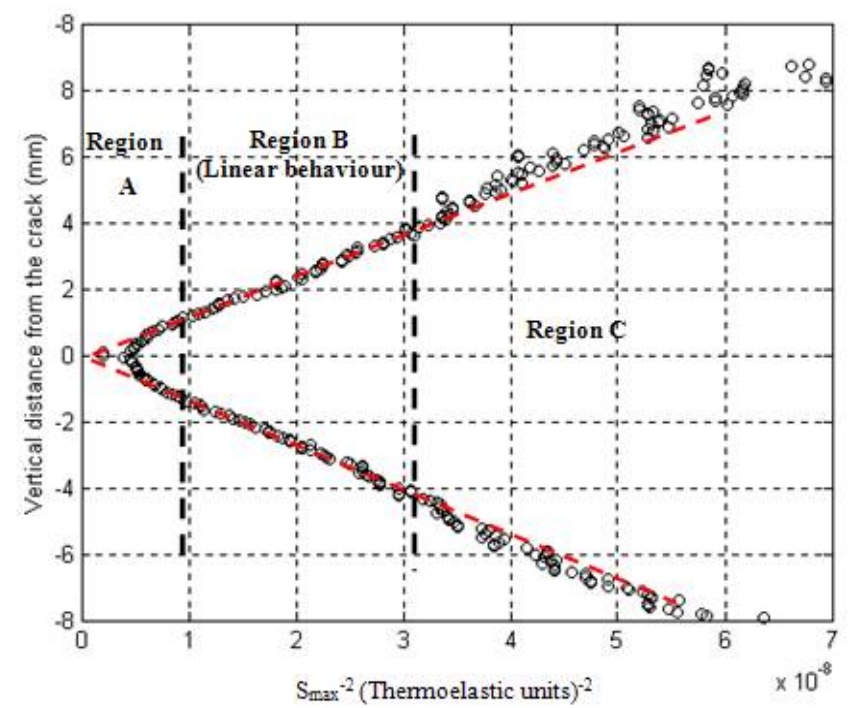

Figure 3: Graph of showing the linear relationship between the vertical distance from the crack tip and $\left(\mathrm{S}_{\max }\right)^{-2}$ employed in the methodology of Stanley and Chan for the calculation of the stress intensity factor from thermoelastic data.

Based on previous analyses, the region of validity for the model is identified in (Figure 3) as the portion of the graph where a linear relationship is observed (Region B). Consequently, data is collected from the corresponding region of the thermoelastic image where such a linear relationship in Stanley's plot exists. The collected points are used to fit the stress field equation described by Muskhelishvili's model and to reconstruct the stress field around the crack tip. From the resultant fitting equation the SIF can be inferred (figure 4). 


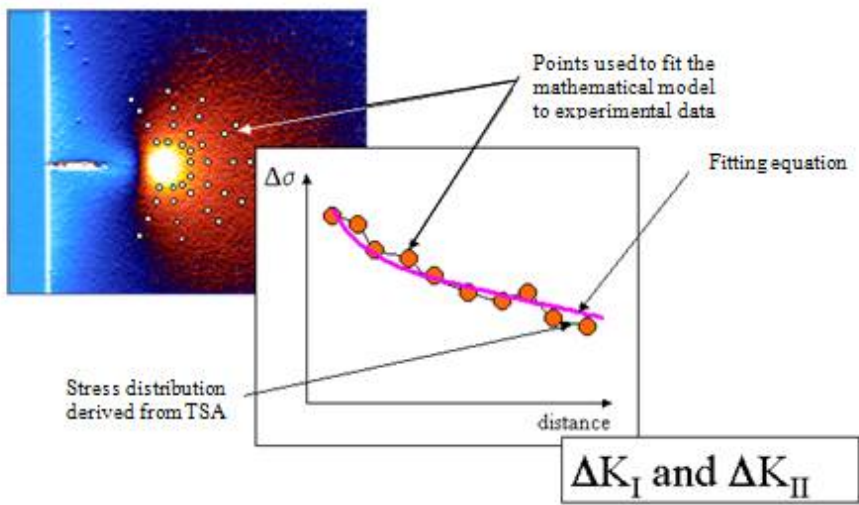

Figure 4: Schematic illustration of the methodology for calculating the stress intensity factor from thermoelastic data.

\section{EXPERIMENTAL SET-UP}

7 o support the idea that TSA can provide accurate information about the real fatigue crack driving force a fatigue test was conducted using aluminium 2024 CT specimens (Figure 5.A). The specimen was initially prepared by bonding three strain gauges (Tokyo Sokki Kenkyujo Co., Ltd., type FPA-2-11, $2 \mathrm{~mm}, 120 \pm 0.5 \Omega$ ) at the different locations. Two of the strain gauges were located on the specimen's surface at $62 \mathrm{~mm}$ from the edge of the specimen in line with the notch. One of them was aligned with the notch direction while the other was inclined $45^{\circ}$ respect to the notch line (Figure 5.B). The third strain gauge was located at the middle part of the back of the specimen (Figure 5.B).

A)

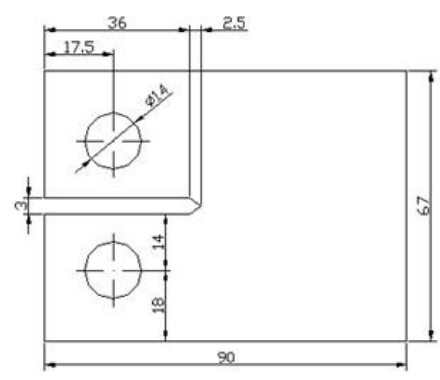

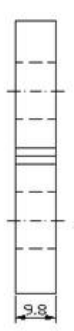

Figure 5: A) Illustration the dimension of aluminium CT specimens employed for fatigue tests; B) Scheme showing the location and orientation of the strain gauges employed for the calculation of the opening and closing loads from the compliance traces

The specimen was initially pre-cracked and a crack was initiated and grown until it was approximately $3 \mathrm{~mm}$ from the two surface strain gauges (42 mm crack length). Subsequently, increasing load steps were gradually applied with the aim of increasing the R-ratio. For each load step, several load and strain readings were collected over a period of $0.2 \mathrm{~s}$ using the three strain gauges. The collected data files were processed and the opening and closing loads estimated using the strain offset technique.

At the same time, thermoelastic images corresponding to the crack at the different load steps were captured at the front of the specimen. $\Delta K$ results from TSA were compared to the nominal $\Delta K$ [10] for the R-ratios achieved by the increasing applied load steps.

$$
\begin{aligned}
& \Delta \mathrm{K}_{\text {nom }}=\frac{\Delta \operatorname{Pf}(\alpha)}{\mathrm{t} \sqrt{\mathrm{W}}}, \quad \alpha=\frac{\mathrm{a}}{\mathrm{W}} \\
& \mathrm{f}(\alpha)=\frac{2+\alpha}{(1-\alpha)^{3 / 2}}\left(0.886+4.64 \alpha-13.32 \alpha^{2}+14.72 \alpha^{3}-5.6 \alpha^{4}\right)
\end{aligned}
$$

Where $\Delta P$ is the load range, $a$ is the crack length and $t$ and $W$ are the specimen thickness and width respectively. 


\section{CALCULATION OF THE OPENING LOAD FROM THE ANALYSIS OF THE COMPLIANCE TRACES}

7 he hardware employed consisted of a PC (Viglen-Pentium $200 \mathrm{MHz}$ Intel processor) provided with an enhanced multifunction I/O PCI board (National Instruments model PCI 6052E) connected to a BNC accessory (National Instrument model BNC-2090). The system was controlled using Lab View software version 6.0 and made it possible to store load and strain data corresponding to a loading-unloading cycle as ASCII files.

Since data were affected by random noise, it was necessary to employ a noise reduction technique. The technique adopted was an incremental polynomial method similar to the recommended ASTM method for data reduction in fatigue tests [11]. Subsequently, for the calculation of the opening/closing loads the strain offset technique was adopted [12]. Initially, data was divided into two sets according to the loading and the unloading branches and plotted as load versus strain plots. The unloading branch was first employed to determine the specimen compliance in the absence of closure by fitting a first order polynomial to its upper part. After that, the fitted first order polynomial was employed to perform the strain-offset calculation, which consisted of subtracting the actual strain data (affected by closure) from the fitted equation (not affected by closure). Subsequently, the load against strain-offset was plotted to estimate the closing load. The closing load was then calculated as the points where data in the plot last crossed the zero strain offset (Figure 6.A).

a)

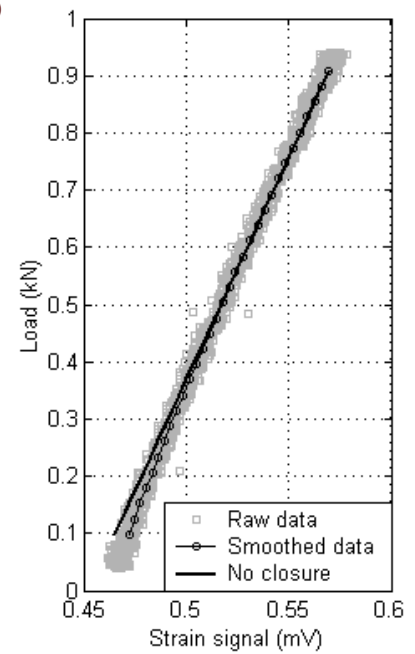

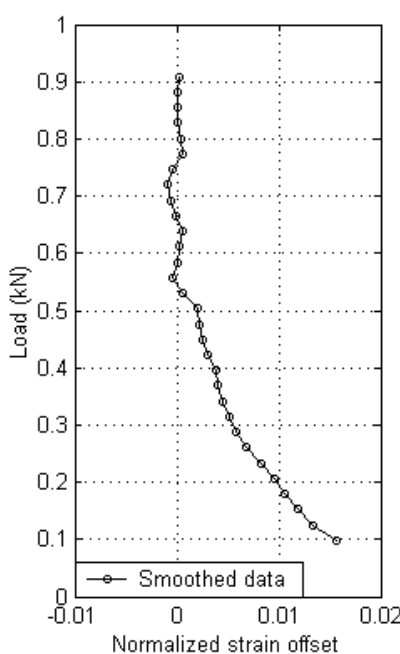

b)

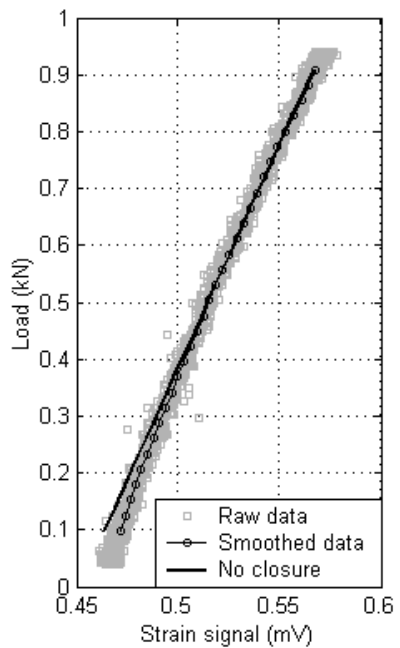

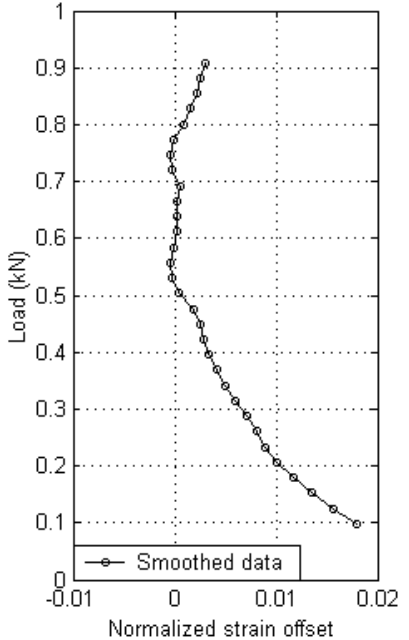

Figure 6: Illustration showing load vs. strain and load vs. strain-offset plots for a $42 \mathrm{~mm}$ crack in an aluminium CT specimen loaded between 0.94 and $0.04 \mathrm{kN}$ (R-ratio 0.04). a) Unloading branch. b) Loading branch.

For the loading branch an identical methodology was followed. However, in this case the strain-offset calculation was performed using the same fitting first order polynomial obtained for the unloading branch. Finally, the opening load was calculated following the same criteria as previously adopted for the unloading branch (Figure 6.B). Results corresponding to the opening and closing loads estimated from data at the different locations (horizontal, $45^{\circ}$ and back face) are presented in figures 7.B, 7.B and 7.C.
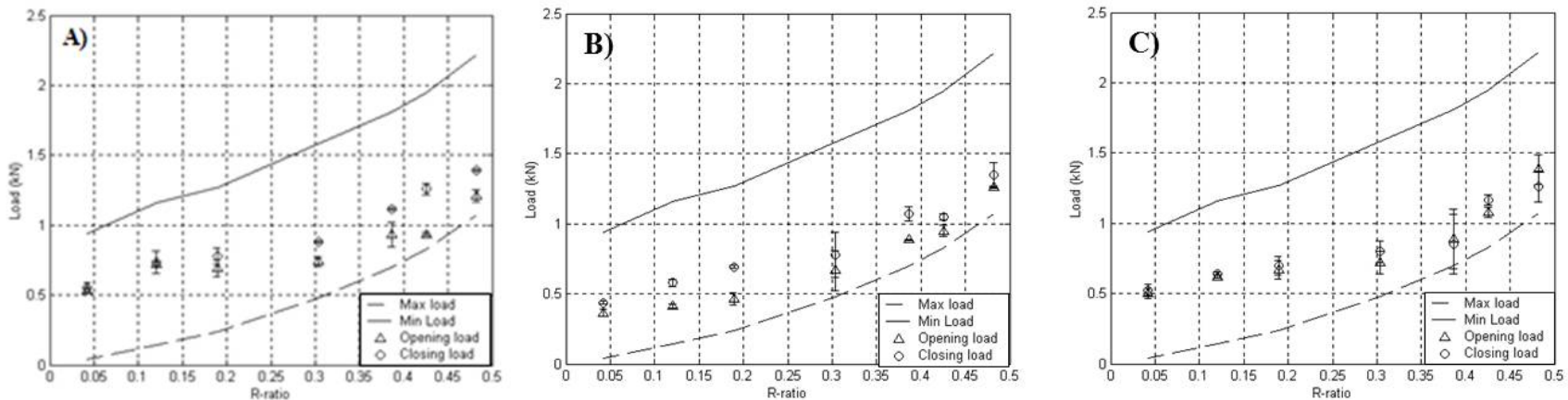

Figure 7: A) Illustration showing the variation of the opening and closing loads with the R-ratio for a 42 mm crack using a horizontal strain gauge, B) a $45^{\circ}$ strain gauge, C) a back face strain gauge. 


\section{TSA RESULTS}

$\mathrm{T}$

o proceed to a direct comparison of thermoelastic results with those previously obtained from the analysis of the compliance traces, $\Delta K$ values inferred from TSA (figure 8.A) were also employed to calculate an equivalent opening/closing load according to equation 5. As for the case of compliance-based techniques, the equivalent opening load was plotted for the R-ratios corresponding to every load step and compared to the maximum and minimum applied loads. Results are presented in figure 8.B.
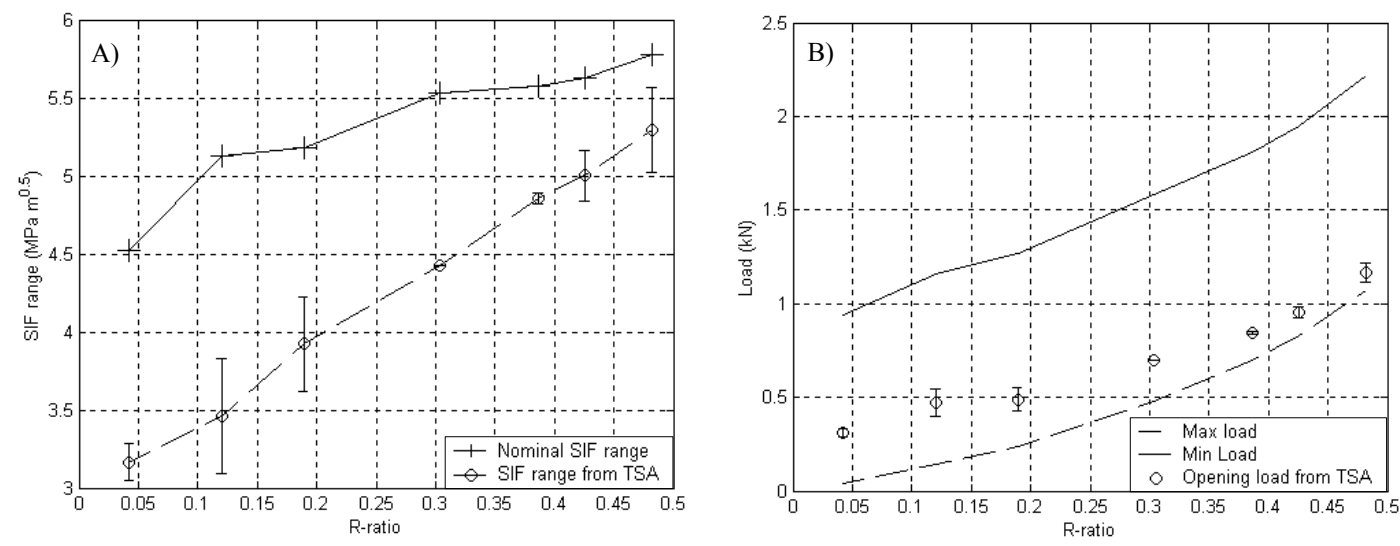

Figure 8: A) Plot showing the variation of $\Delta \mathrm{K}$ with the $\mathrm{R}$-ratio for a sequence of thermoelastic images corresponding to a $42 \mathrm{~mm}$ crack. B) Plot illustrating the variation with the R-ratio of the opening load inferred from a sequence of thermoelastic images.

\section{DISCUSSION}

7 he initial aspect investigated was the repeatability of the results based on compliance measurements when using strain gauges bonded at the three locations when applying increasing R-ratio steps. Results for the opening/closing were observed in all the cases to give similar results, with slightly smaller values for the case of the $45^{\circ}$ strain gauge (figures7.A, B and C). In addition, it was also observed that as the R-ratio increased from 0.04 to 0.3 , the opening/closing loads remained relatively constant and in all the cases with values higher than the minimum load. This clearly showed the presence of closure at low R-ratios. However, as the R-ratio increased from 0.3 upwards, the opening/closing load was observed to follow the minimum load indicating a considerably reduction in the closure levels.

To check the ability of TSA to successfully infer the effective $\Delta K$, thermoelastic images were captured at the same $R$-ratio steps simultaneously with compliance experiments. Results for $\Delta K$ obtained from the analysis of the thermoelastic images (Fig. 8.A) show the same behaviour as observed in the compliance analysis. As the R-ratio increases the $\Delta K$ from TSA tends to approach the nominal $\Delta K$, showing a reduction in the closure level as the $\mathrm{R}$-ratio increases. Moreover, for direct comparison of thermoelastic results with those previously obtained using the strain-offset technique an equivalent opening/closing load was inferred from thermoelastic values of $\Delta K$ using equation 5 (Fig. 8.B). Results were observed to follow the same tendency as previous results obtained using the strain-offset technique for increasing $R$-ratio with very similar values to those obtained using the $45^{\circ}$ strain gauge. This issue clearly support the ability of TSA to measure the effective $\Delta K$ rather that the nominal $\Delta K$.

\section{CONCLUSIONS}

SA has been presented as a novel technique to quantify the effective stress intensity factor range during cyclic loading. Results from TSA have been compared with those obtained using a standard technique for crack closure measurement namely, the strain-offset technique. Compliance results have clearly shown the presence of crack 
closure at low R-ratios in the analysed samples. Moreover, results inferred from the analysis of thermoelastic images have been observed to be in good agreement with those obtained using the strain offset technique, showing the ability of TSA to successfully quantify the effective $\Delta K$.

\section{REFERENCES}

[1] James, M.N., Some unresolved issues with fatigue crack closure measurement, mechanisms and interpretation problems, Advance in Fracture Research, Proceedings of the ninth International Conference on Fracture. Edited by B.L. Karihaloo et al., Pergamon Press, 5 (1996) 2403-14.

[2] Díaz, F.A., Yates, J.R., Patterson, E.A., Some improvements in the analysis of fatigue cracks using thermoelasticity, Int. J. Fatigue, 26, 4 (2004) 365-376.

[3] Díaz, F.A., Patterson, E.A., Tomlinson R.A., Yates, J.R., Measuring stress intensity factors during fatigue crack growth using thermoelasticity, Fat. Fract. Eng. Mat. Struct., 27 (2004) 571-584.

[4] Thomson, W. (Lord Kelvin), On the Thermoelastic, Thermomagnetic and Pyro-electric Properties of Matters, Philosophical Magazine, 5 (1878) 4-27.

[5] Dulieu-Smith, S.M., Alternative calibration techniques for quantitative thermoelastic stress analysis, Strain, 31 (1995) 9-16.

[6] Sanford, R., Dally, J.W., A general method for determining mixed-mode stress intensity factors from isochromatic fringe pattern, Engineering Fracture Mechanics, 11 (1979) 621-633.

[7] Muskhelishvili, N.I., Some basic problems of the mathematical theory of elasticity, Third edition, Noordhoff Ltd., Groningen, Holland, (1953).

[8] Stanley, P., Chan, W.K., Mode II crack studies using the "SPATE" technique', Proc. of SEM spring conference on experimental mechanics, New Orleans, USA, (1986) 916-923.

[9] Westergaard, H.M., Bearing pressures and cracks, J. of Applied Mechanics, 6 (1939) A49-63.

[10] Murakami, Y., Stress Intensity Factors Handbook, Pergamon, Oxford, (1987).

[11] ASTM, E647-95a, American Society of Testing and Materials, 03.01 (1999).

[12] Skorupa, M., Beretta, S., Carboni, M., Machniewicz, T., An algorithm for evaluating crack closure from local compliance measurements, Fat. Fract. Eng. Mat. Struct., 25 (2002) 261-273. 